Journal of Thermal Engineering, Vol. 7, No. 4, pp. 1016-1029, May, 2021

Yildiz Technical University Press, Istanbul, Turkey

\title{
NUMERICAL SIMULATION OF PRANDTL NUMBER EFFECT ON ENTROPY GENERATION IN A SQUARE CAVITY
}

\author{
Nawal Ferroudj ${ }^{1, *}$, Hasan Köten ${ }^{2}$, Sacia Kachi ${ }^{3}$, Saadoun Boudebous ${ }^{4}$
}

\begin{abstract}
In this study, entropy generation in laminar mixed convection in a square fluid-filled cavity is numerically studied. The middle of the lower wall of the cavity is heated to a constant temperature Th while the side-walls are maintained at a constant temperature Tc, and moving upper ward at a constant velocity to understand the effects of irreversibility distribution on the entropy generation for different engineering applications. For the studied control surface, remaining parts of lower and upper walls are adiabatic. The finite difference method is used to solve the governing equation. The entropy analysis is carried out to determine the irreversibility which is generated in the cavity for different Prandtl number $(\mathrm{Pr}=0.0212,0.71$ and 6.35), the effect of the irreversibility distribution ratio on entropy generation was investigated. It seen that effect of irreversibility distribution ratio $\left(\gamma=10^{-2}\right.$ and $\left.10^{-4}\right)$ have play important role on the total entropy for different Prandtl number. Also it is clear that, for all Prandtl number, the total entropy generation increase by increasing the irreversibility distribution ratio and the increase of Prandtl number regardless the values of $\mathrm{Ri}=1$ and $\mathrm{Re}=100$ because of the increase of the velocity gradients.
\end{abstract}

\section{Keywords: Generation Entropy Generation, Prandtl Number, Square Cavity, Finite Difference Method}

\section{INTRODUCTION}

Flow and heat transfer in different cavities have a wide range of applications in the engineering devices and industry, such as: the solar energy system, cooling systems producing nuclear energy, chemical and electronic devices and heat exchangers etc [1-3]. Due to its uttermost importance, many applications, many researches, investigations and studies have been created to raise the performance and quality of those engineering systems. Among these studies is the study of the entropy generation. Entropy generation is a measure of dissipated energy and degradation of the performance of operational devices, such as transport and flow process [1]. The study of entropy generation has been initiated by Bejan [3], which considers that the entropy generation of fluid flow under forced and laminar convection is induced by viscous and thermal mechanisms, predicting (involving) velocity and temperature gradients. We present in the following some studies concerning this subject. Yilbas et al. [4] presented a numerical study of the entropy generation in natural convection and laminar flow in a square cavity with bottom-heated, topdown and vertical walls adiabatic. Nine cases were studied by varying the difference of temperature between the hot wall and the cold wall (the Rayleigh number (Ra) varies between $1.3610^{5}$ and $3.9610^{5}$ ). They found that entropy generation increases as the velocity of flow in the horizontal direction increases. The minimum of the entropy generation is raised for a particular number of Rayleigh numbers (Ra). Baytas et al. [5] reported a numerical study of the entropy generation in laminar natural convection in an inclined porous square cavity. Simulations are performed for angles of inclination varying between $0^{\circ}$ and $360^{\circ}$ and Rayleigh number values (Ra) varying between $10^{2}$ and $10^{4}$. They found that thermal irreversibilities dominate viscous irreversibilities as the Rayleigh number (Ra) increases. The Bejan number (Be) increases significantly between $\varphi=150^{\circ}$ and $\varphi=270^{\circ}$ (angles of inclination). Shuja et al. [6] conducted a numerical study of the entropy generation in laminar mixed convection in a square cavity, containing an obstacle. The influence of the location of the fluid outlet and the aspect ratio $(\mathrm{a}=0.25$ and 4$)$ of the obstacle were examined. Yilbas et al. [7] have taken over the same study as the previous one with the same geometric configuration. In this second study, the influence of the Stanton number and the aspect ratio $(a=0.25, a=$

This paper was recommended for publication in revised form by Regional Editor Younes Menni

${ }^{1}$ Laboratory of Biotechnology, National Higher School of Biotechnology, Taoufik Khaznadar, University town, Constantine, Algeria

${ }^{2}$ Department of Mechanical Engineering, Istanbul Medeniyet University, Istanbul, Turkey

${ }^{3}$ Faculty of Process Engineering, University of Salah Boubnider Constantine 3, Algeria

${ }^{4}$ Faculty of Sciences and Applied Sciences, University of Larbi BenM'hidi, Oum el Bouaghi, Algeria

*E-mail address: ferroudj.nawal@gmail.com,n.ferroudj@ensbiotech.edu.dz

Orcid id: 0000-0001-7743-0971, 0000-0002-1907-9420, 0000-0003-4420-4331, 0000-0003-0136-0748

Manuscript Received 28 October 2020, Accepted 12 February 2021 
1 , and $\mathrm{a}=4$ ) of the obstacle were examined. Magherbi et al. [8] conducted a numerical study on the entropy generation of unsteady natural convection in a square cavity. The Rayleigh number (Ra) varies between $10^{3}$ and $10^{5}$, whereas the ratio of the irreversibility distribution $(\varphi)$ varies between $10^{-4}$ and $10^{-1}$. The evolution of the maximum entropy generation in this configuration has been reported. The effect of the ratio of irreversibility distribution was examined. Ovando-Chacon et al. [9] studied numerically the mixed convection in a square cavity heated from corners. They found that the entropy generation by heat transfer becomes more intense at the vertical walls and the central low part of the cavity due to the stronger temperature gradients at this region. Bouchoucha et al. [10] presented an investigation of the natural convection and entropy generation of nanofluids in a square cavity. They observed that the average Nusselt number ratio increases with the solid volume fraction, and the maximum total entropy generation ratio occurs at a low Rayleigh number for different values of $\phi$. Selimefendigil et al. [11] examined the mixed convection and entropy generation in a vented cavity with inlet and outlet ports under the effects of an inclined magnetic field. The numerical simulations are performed for various values of Reynolds numbers between $100<\mathrm{Ra}<500$, Hartmann number between 0 and 50, and solid particle volume fractions of $\mathrm{CuO}$ nanoparticles between 0 and $4 \%$. They found that the addition of nanoparticles increases the overall entropy generation rate. The literature review shows that the entropy generation in laminar mixed convection in cavities is a subject that remains to be explored. Different studies were studied and reported in the literature [12-16].

The present work investigates the entropy generation of mixed laminar convection in a square cavity. The main objective is to determinate the effect of Prandtl number on the fields of streamlines, isotherm, heat and viscous entropy generation and Bejan number, evolution of heat and viscous entropy generation-profiles, Bejan end Nusselt number and total entropy generation- profiles.

\section{MATERIAL AND METHODS \\ Problem Statement}

The physical model considered is shown in Fig.1. It is a square cavity the bottom of which a part is subjected to a constant temperature, of length equal to four fifth of the cavity L. the side walls of this cavity move upwardly with a specified velocity, and the all other parts are considered adiabatic. The entropy of the fluid flow is generated within the cavity created 2 by the irreversibility of the viscous and thermal mechanisms due to velocity and temperature gradients.

It is assumed that the fluid in the cavity is Newtonian, incompressible, and has constant properties, for laminar flow, neglecting the viscous dissipation effect, and employing the Boussinesq approximation.

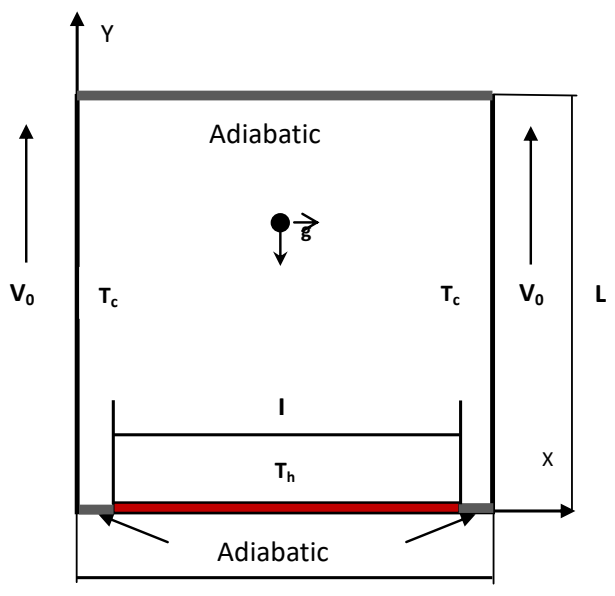

$\mathbf{L}$

Figure 1. Physical model 


\section{Dimensionless Governing Equations}

The governing equations for the present problem for steady two-dimensional mixed convection flow in a square cavity are the conservation continuity of mass, momentum and energy equation than can be expressed as follows [17]:

- Continuity equation:

$$
\frac{\partial u}{\partial x}+\frac{\partial v}{\partial y}=0
$$

- Momentum equation:

$$
\begin{gathered}
\frac{\partial u}{\partial t}+u \frac{\partial u}{\partial x}+v \frac{\partial u}{\partial y}=-\frac{1}{\rho} \frac{\partial P^{*}}{\partial x}+v\left(\frac{\partial^{2} u}{\partial x^{2}}+\frac{\partial^{2} u}{\partial y^{2}}\right) \\
\frac{\partial v}{\partial t}+u \frac{\partial v}{\partial x}+v \frac{\partial v}{\partial y}=-\frac{1}{\rho} \frac{\partial P^{*}}{\partial y}+v\left(\frac{\partial^{2} v}{\partial x^{2}}+\frac{\partial^{2} v}{\partial y^{2}}\right)+g \beta\left(T-T_{f}\right)
\end{gathered}
$$

- Energy equation:

$$
\frac{\partial T}{\partial t}+u \frac{\partial T}{\partial x}+v \frac{\partial T}{\partial y}=\alpha\left(\frac{\partial^{2} T}{\partial x^{2}}+\frac{\partial^{2} T}{\partial y^{2}}\right)
$$

It is assumed that the fluid in the cavity is Newtonian, incompressible, and has constant properties, for laminar flow, neglecting the viscous dissipation effect, and employing the Boussinesq approximation.

The dimensionless form of the governing equations may be written with following dimensionless variables of numbers:

$$
X=\frac{x}{L}, Y=\frac{y}{L}, \quad \theta=\frac{T-T_{c}}{T_{H}-T_{c}}, U=\frac{u}{V_{0}}, \quad \mathrm{~V}=\frac{v}{V_{0}}, P=\frac{p-\rho g y}{\rho V_{0}^{2}}, \tau=t \frac{V_{0}}{L}
$$

The governing equations in dimensionless form are transformed into stream-vorticity function $(\omega, \psi)$ and energy equation, are expressed as follows:

- Energy equation

$$
\frac{\partial \theta}{\partial \tau}+U \frac{\partial \theta}{\partial X}+V \frac{\partial \theta}{\partial Y}=\frac{1}{\operatorname{RePr}}\left(\frac{\partial^{2} \theta}{\partial X^{2}}+\frac{\partial^{2} \theta}{\partial Y^{2}}\right)
$$

- Stream function equation

$$
\frac{\partial^{2} \psi}{\partial X^{2}}+\frac{\partial^{2} \psi}{\partial Y^{2}}=-\omega
$$

- Vorticity equation

$$
\frac{\partial \omega}{\partial \tau}+U \frac{\partial \omega}{\partial X}+V \frac{\partial \omega}{\partial Y}=\frac{1}{\operatorname{Re}}\left(\frac{\partial^{2} \omega}{\partial X^{2}}+\frac{\partial^{2} \omega}{\partial Y^{2}}\right)+R i \frac{\partial \theta}{\partial X}
$$


Reynolds (Re), Prandtl $(\mathrm{Pr})$ and Grashof $(\mathrm{Gr})$ are the principal controls parameters; they are defined as follow:

$$
\operatorname{Re}=\frac{V_{0} L}{v} \quad, \quad \operatorname{Pr}=\frac{v}{\alpha} \quad, \quad G r=\frac{g \beta \Delta T L^{3}}{v^{2}}
$$

- Components of the velocity:

$$
U=\frac{\partial \psi}{\partial Y} \quad, \quad V=-\frac{\partial \psi}{\partial X}
$$

The dimensionless vorticity and stream are defined by:

$$
\begin{gathered}
\omega=\frac{\partial V}{\partial X}-\frac{\partial U}{\partial Y} \\
\frac{\partial^{2} \psi}{\partial X^{2}}+\frac{\partial^{2} \psi}{\partial Y^{2}}=-\omega
\end{gathered}
$$

The local Nusselt number $\mathrm{Nu}(\mathrm{X})$ is used to characterize the heat flux between the heated part of the bottom wall, and the fluid in the cavity. It is expressed as [18]:

$$
N u(X)=-\left.\frac{\partial \theta}{\partial Y}\right|_{Y=0}
$$

The average Nusselt number is obtained by integration the previous local Nusselt number $\mathrm{Nu}(\mathrm{X})$ :

$$
N u_{m}=\int_{0}^{L} N u(X) d X
$$

- Entropy generation equation

Entropy generation rate for a two-dimensional flow is expressed as follow [19, 20]:

$$
s_{g e n}=\frac{k}{T^{2}}\left[\left(\frac{\partial T}{\partial x}\right)^{2}+\left(\frac{\partial T}{\partial y}\right)^{2}\right]+\frac{\mu}{T}\left\{2\left[\left(\frac{\partial u}{\partial x}\right)^{2}+\left(\frac{\partial v}{\partial y}\right)^{2}\right]+\left(\frac{\partial u}{\partial y}+\frac{\partial v}{\partial x}\right)^{2}\right\}
$$

By dividing $\mathrm{s}_{\text {gen }}$ by $\frac{k \Delta T^{2}}{(T L)^{2}}$ and by using the same dimensionless parameters, the dimensionless local entropy generation due to heat transfer and fluid friction for a two dimensional heat and fluid flow in Cartesian coordinates can be written as:

$$
\dot{S}_{\text {gen }}=\underbrace{\left[\left(\frac{\partial \theta}{\partial X}\right)^{2}+\left(\frac{\partial \theta}{\partial Y}\right)^{2}\right]}_{S_{\theta}}+\underbrace{\gamma\left[2\left\{\left(\frac{\partial U}{\partial X}\right)^{2}+\left(\frac{\partial V}{\partial Y}\right)^{2}\right\}+\left(\frac{\partial U}{\partial Y}+\frac{\partial V}{\partial X}\right)^{2}\right]}_{S_{\psi}}
$$

where $\gamma$ is the irreversibility distribution ratio is given as 


$$
\gamma=\frac{\mu T V_{o}^{2}}{k \Delta T^{2}}
$$

The first and the second terms $\left(S_{\theta}, S_{\psi}\right)$ are represented the local entropy generation due to the heat transfer and viscous effects, respectively.

The total entropy generation is expressed by:

$$
S_{g e n}=\int_{A} \dot{S}_{g e n}(X, Y) d X d Y
$$

The Bejan number $\mathrm{Be}$ is defined as the ratio between entropy generations due to heat transfer irreversibility to the total entropy generation, it's written as:

$$
B e=\frac{S_{\theta}}{S_{\theta}+S_{\psi}} \quad, \quad\left\{\begin{array}{l}
S_{\theta}=\int_{A} \dot{S_{\theta}} d A \\
S_{\psi}=\int_{A} \dot{S_{\psi}} d A
\end{array}\right.
$$

It is appropriate to mention average Bejan number in order to determinate which is the dominant, heat transfer or fluid friction irreversibility.

Therefore, Be $>0.5$ implies dominance of heat transfer irreversibility, and $\mathrm{Be}<0.5$ implies dominance of fluid friction irreversibility.

\section{Dimensionless Boundary Conditions}

The boundary conditions which are adopted for the resolution of the problem are:

$$
\begin{aligned}
& \theta=0 \quad U=0, V=1 \text { for } X=0 \text { and } 1 \text { and } 0<Y<1 \\
& \theta=1 \quad U=V=0 \quad \text { for } \quad Y=0 \quad \text { and } \quad \frac{1-\varepsilon}{2} \leq X \leq \frac{1+\varepsilon}{2} \\
& \frac{\partial \theta}{\partial Y}=0 \quad U=V=0 \quad \text { for } \quad Y=0 \quad \text { and } \quad 0<X<\frac{1-\varepsilon}{2}, \frac{1+\varepsilon}{2}<X<1 \\
& \frac{\partial \theta}{\partial Y}=0 \quad U=V=0 \quad \text { for } \quad Y=1 \quad \text { and } \quad 0<X<1
\end{aligned}
$$

In which $\varepsilon=\frac{l}{L}$ is the dimensionless length of the heated part.

\section{NUMERICAL METHOD}

The dimensionless equations are solved by a numerical approach on the finite difference method. The system of equations (5)-(14), together with the initial and boundary conditions, stated above were discredited of the various terms of the governing equations is developed using high order scheme as the third order upwind scheme for the convective terms [21], centred fourth order for the diffusive terms, as well as the partial derivatives of the first order. The Runge-Kutta fourth order used to discredit the temporal term scheme [22]. An iterative procedure based on successive Non Linear Over Relaxation method (NLOR) was used to solve the discredited stream function equation [23]. There are different studies related the applications in the literature [24-29]. A convergence criterion for the stream function was imposed as each time step. The computation is carried out using a developed FORTRAN code. In order to convergence the obtained numerical results, total entropy generation for square cavities with different $\phi$ and Ra values were obtained and compared with the results of similar studies. Results are agreement with the literature. 


\section{GRID INDEPENDENCE STUDY}

The effect of grid size is examined on four different grids $81 \times 81 ; 101 \times 101 ; 161 \times 161$ and $201 \times 201$. These comparisons allow us the choice of mesh $101 \times 101$ for the present work, as it provides a good compromise between the accuracy of these calculations and the duration of computation time.

Table 1. Maximum values of Num for different grids, at $\mathrm{Ri}=17$

\begin{tabular}{|c|c|c|}
\hline Grid & $\begin{array}{c}\text { Time } \\
{[\mathbf{s e c}]}\end{array}$ & $\begin{array}{c}\text { Average Nusselt Number: } \\
\mathbf{N u m}\end{array}$ \\
\hline $81 \times 81$ & 5425 & 5.52864 \\
\hline $101 \times 101$ & 7248 & 5.55646 \\
\hline $161 \times 161$ & 9653 & 5.61192 \\
\hline $201 \times 201$ & 13264 & 5.63508 \\
\hline
\end{tabular}

\section{CODE VALIDATION}

In order to check on the validity of the computer code developed for the solution of the problem considered in the present study, it was validated with Aydin and Yang [30]. Figure 2 illustrates a comparison between the obtained results and those obtain in the Aydin's results. The results are presented in terms of dimensionless vertical velocity profiles $\mathrm{V}$, at the horizontal mid-plane. The figures show excellent agreement between the present code and their results.

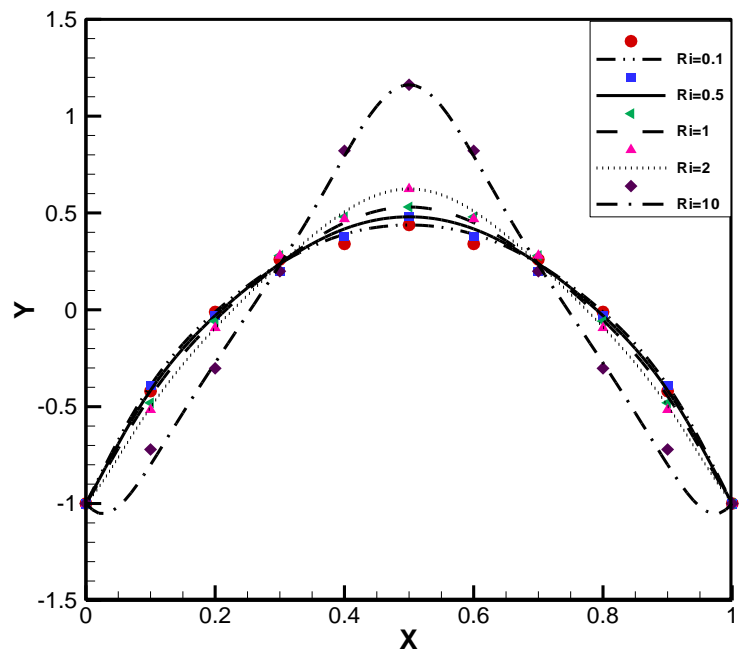

Figure 2. Comparison of the horizontal mid-plane velocity component at $\mathrm{Y}=0.5$ with the results obtained in Aydin and yang (Lines: Present work, Symbols: Aydin's results)

\section{RESULTS AND DISCUSSION}

This investigation is mainly related to entropy generation distribution in a square cavity by using the second law of thermodynamics. Detailed computations have been carried for a fixed Reynolds number $(\operatorname{Re}=100)$, the length of heated part $(1=0.8)$, and for various fluids used in this study are $(\operatorname{Pr}=0.0212,0.71$ and 6.35$)$, within the values of Richardson number and irreversibility distribution ration are $\mathrm{Ri}=1$ and $\gamma=10^{-4}$, respectively. For all the studied grid of size $(101 \times 101)$ with a time steps $\Delta t=10^{-4}$ are found sufficiently enough to achieve the steady state and to realie the imposed convergence criteria mentioned above. 


\section{Streamlines and Isotherms}

Figure 3 illustrates isotherms $(\theta)$ and streamlines $(\psi)$ for $\operatorname{Pr}=0.0212,0.71$ and 6.35. The streamlines represent a symmetrical flow, characterized by a pair of counter-rotating and symmetrical cells for all values of Prandtl number. Thus, the streamlines are concentrated near the upper walls. The general structures of these fields are qualitatively similar, regardless of the Prandtl number.

For low Prandtl number $(\mathrm{Pr}=0.0212)$, the thermal field shows a stratification of temperatures near the heated bottom wall, when Prandtl number increases and reaches the value $\operatorname{Pr}=0.71$ a part of the heat is trained to the upward of the cavity through velocity of the side walls. For high Prandtl number $(\mathrm{Pr}=6.35)$, the isotherm shows that the heat recovered from the hot source is transported by convection to the upwards of the cavity by the pair of center cells.

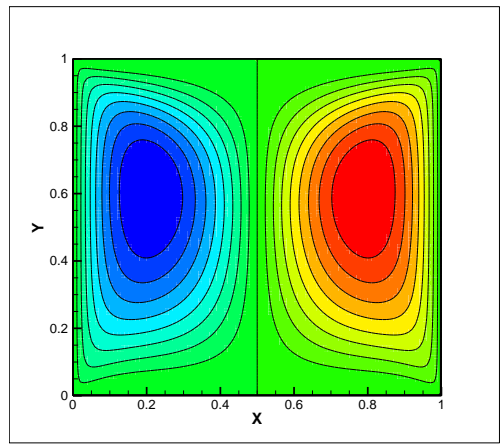

$\operatorname{Pr}=0.0212$

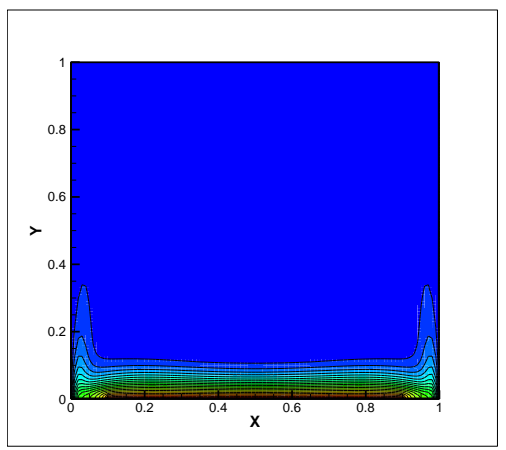

$\operatorname{Pr}=0.0212$

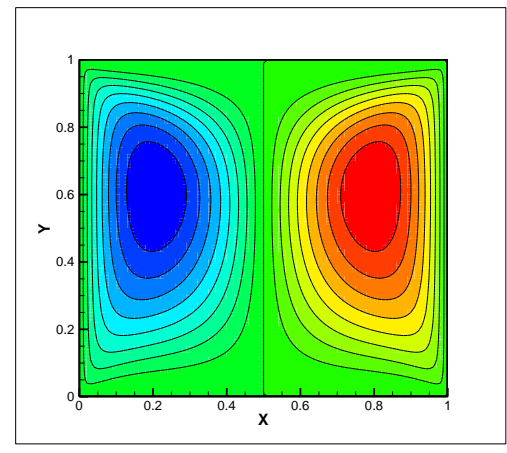

$\operatorname{Pr}=0.71$

a)

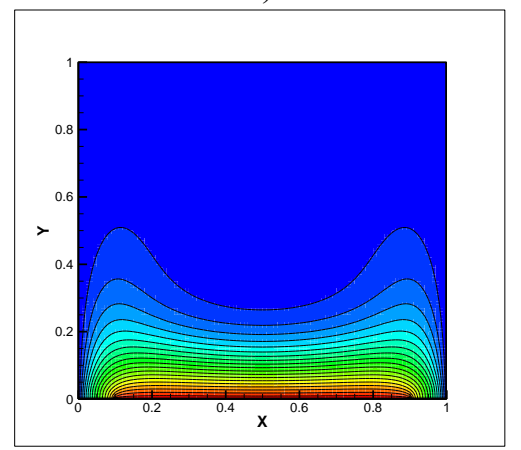

$\operatorname{Pr}=0.71$

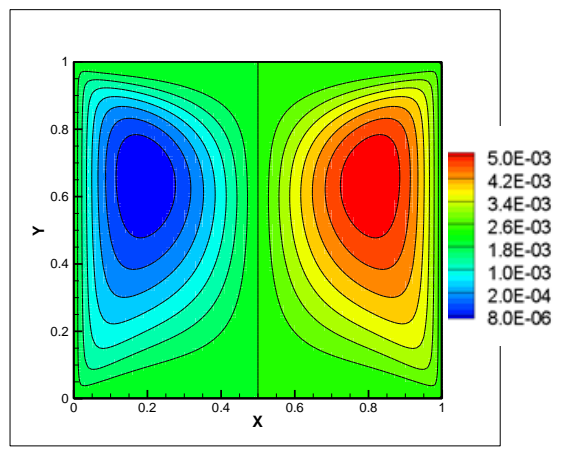

$\operatorname{Pr}=6.35$

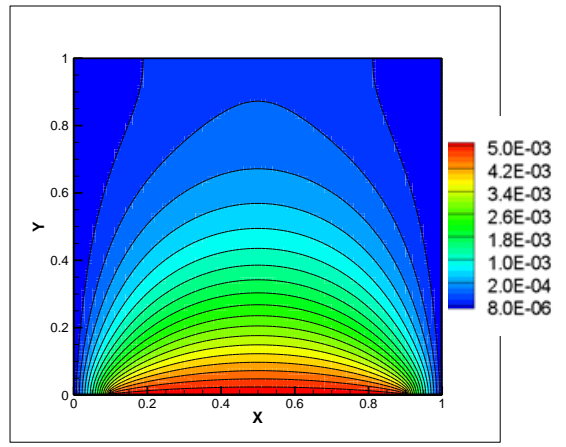

$\operatorname{Pr}=6.35$

b)

Figure 3. Streamlines a) and isotherms b) for different Prandtl numbers, $\mathrm{Ri}=1, \mathrm{Re}=100$ and $\gamma=10^{-4}$

\section{Fields of Heat and Viscous Entropy Generation}

The effect of Prandtl number on the fields of heat and viscous entropy generation present in figure. 4 (a-b), for $\mathrm{Ri}=1$ and $\gamma=10^{-4}$.

The thermal entropy generation is consistent with the temperature distribution; it follows the propagation of heat, given by Figure 3 (a), from the bottom heated part, it is created all along this wall (especially to the extremities of the heated part for the low $\operatorname{Pr}=0.0212$ and 0.71 ). When the Prandtl number increases the heat entropy generation increases, until it is concentrated also along the moving sidewalls for the high Prandtl number $(\operatorname{Pr}=6.35)$.

The viscous entropy generation is created near the two side walls and the four corners of the cavity. Its concentration is decreasing with the increase of the Prandtl number. 
Journal of Thermal Engineering, Research Article, Vol. 7, No. 4, pp. 1016-1029, May, 2021

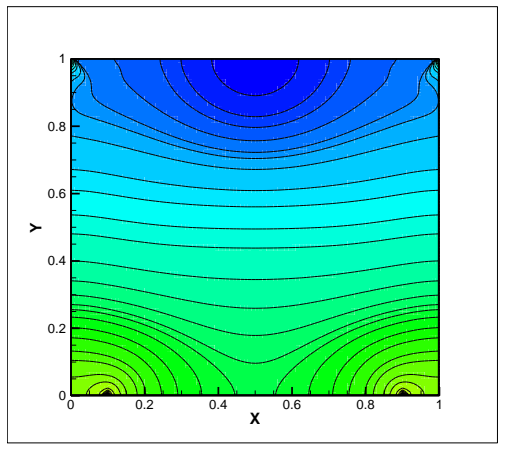

$\operatorname{Pr}=0.0212$

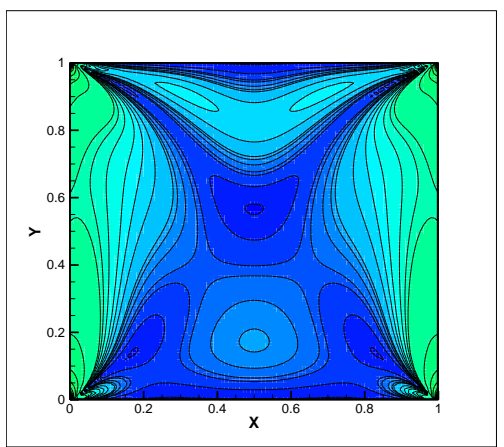

$\operatorname{Pr}=0.0212$

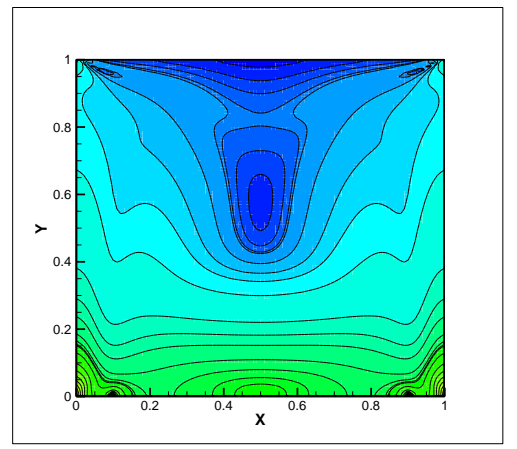

$\operatorname{Pr}=0.71$

a)

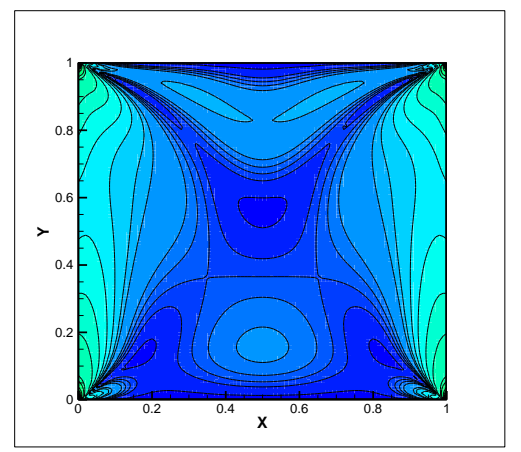

$\operatorname{Pr}=0.71$

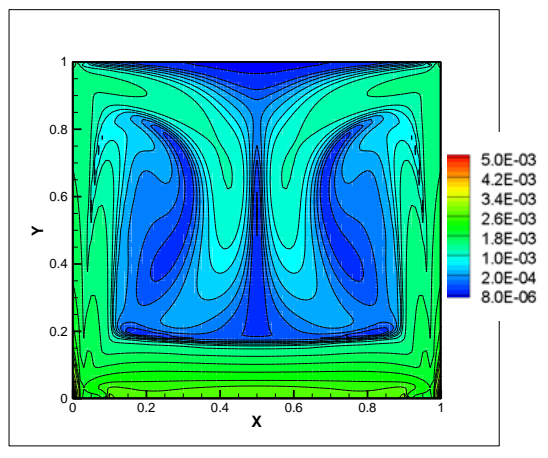

$\operatorname{Pr}=6.35$

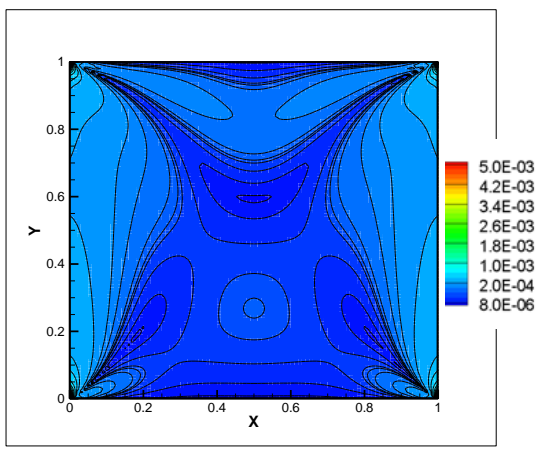

$\operatorname{Pr}=6.35$

b)

Figure 4. Fields of entropy generation due to heat transfer $\mathrm{S}_{\theta}(\mathrm{a})$ and fluid friction $\mathrm{S}_{\psi}(\mathrm{b})$ for different Prandtl numbers, $\mathrm{Ri}=1, \mathrm{Re}=100$ and $\gamma=10^{-4}$

\section{Fields of Bejan Number}

The fields of Bejan number obtained have been presented at three different values of Prandtl number $(\operatorname{Pr}=$ $0.0212,0.71$ and 6.35) for comparison supposes as shown in figure 5. It shows that the Bejan number (development) decreases with the increase in the Prandtl number; it increases until it reaches its maximum value in the lower twothirds and an upper part of the cavity for $\mathrm{Pr}=6.35$. Moreover, the low values of this number are observed on the center of the upper wall and the top of the side walls. These results are confirmed by those obtained in the figures (3$4)$. 


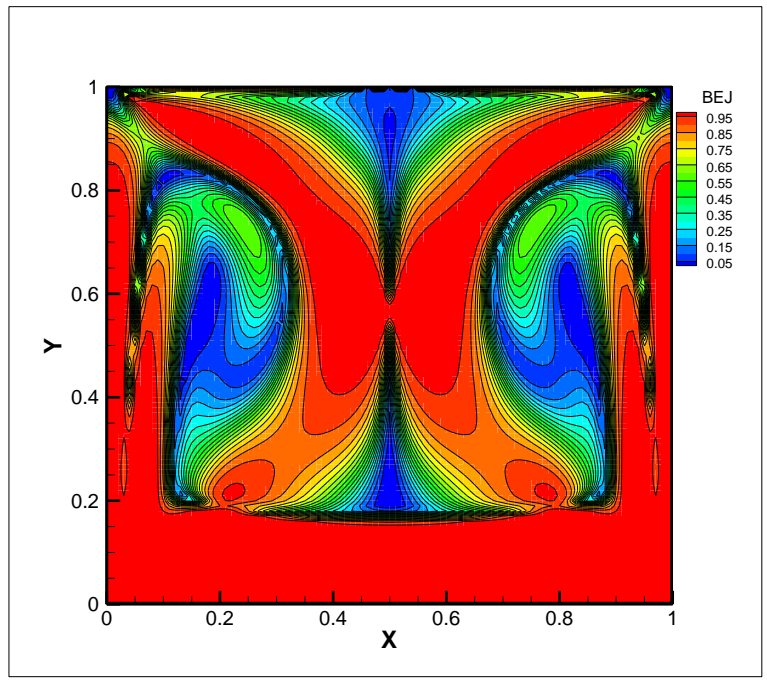

$\operatorname{Pr}=0.0212$

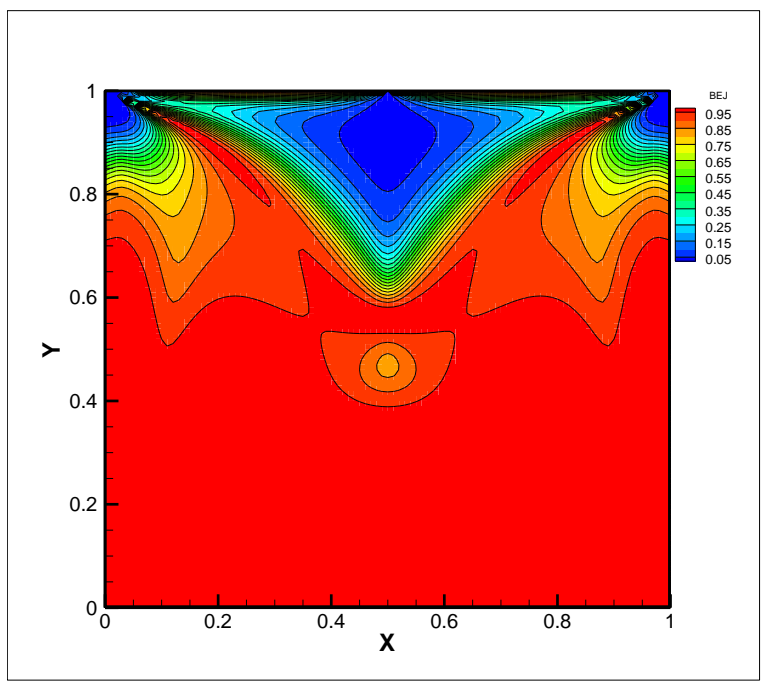

$\operatorname{Pr}=0.71$

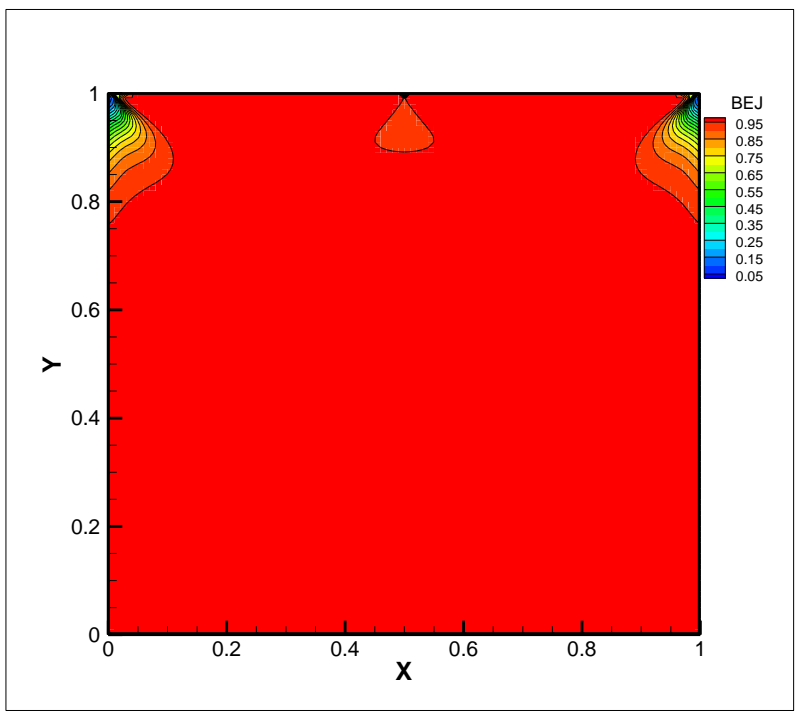

$\operatorname{Pr}=6.35$

Figure 5. Fields of Bejan number for different Prandtl numbers, $\operatorname{Ri}=1, \operatorname{Re}=100$ and $\gamma=10^{-4}$

\section{Profiles of Thermal $\left(S_{\theta}\right)$ and Viscous $\left(S_{\psi}\right)$ Entropy Generation}

In order to investigate the influence of Prandtl number on both local thermal $\left(S_{\theta}\right)$ and viscous $\left(S_{\psi}\right)$ entropy generation, Figure 6 (a-b) illustrates the variation of and with different values of Prandtl number. As it can be seen, for all values of this number, a very rapid decrease then a sudden increase in all the first time, then stabilization towards a constant asymptotic value, has been observed. The increase in the number of Prandtl implies the increase of local thermal entropy generation.

It is observed also that, for all values of Prandtl number, the viscous entropy generation decreases by increasing the Prandtl number regardless the values of Ri and Re. From the value of $\operatorname{Pr}=0.71$, the $S_{\psi}$ varies slightly with the increase of the Prandtl number. We have also observed the low values of $S_{\psi}$ compared to the values of the $\mathrm{S}_{\theta}$, this is confirmed by the results obtained in the figure $4(\mathrm{a}-\mathrm{b})$. 


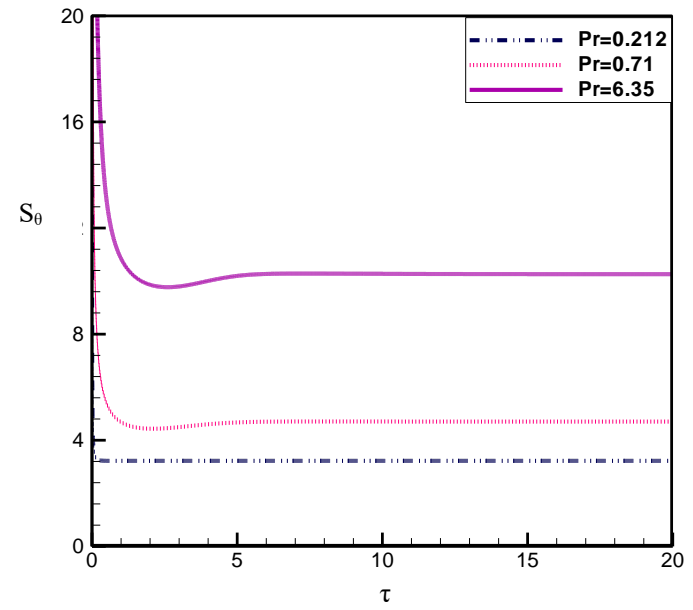

a)

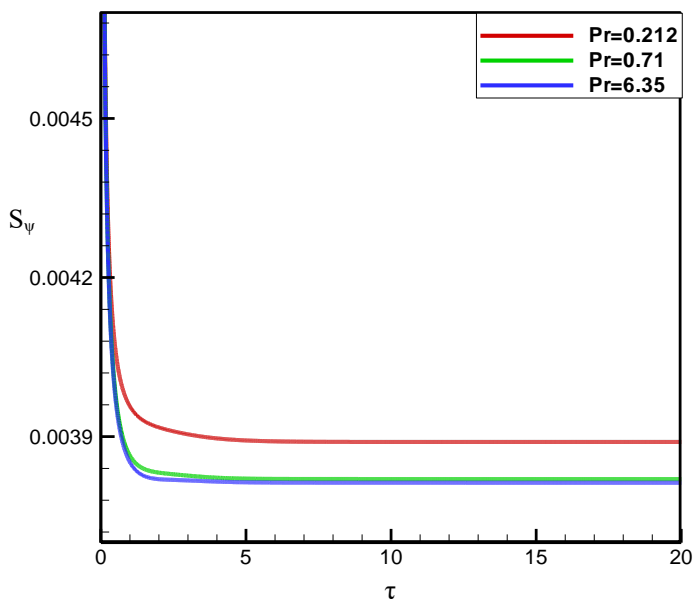

b)

Figure 6. Profiles of entropy generation due to heat transfer $S_{\theta}$ a) and fluid friction $S_{\psi} b$ ) for different Prandtl numbers, $\mathrm{Ri}=1, \mathrm{Re}=100$ and $\gamma=10^{-4}$

\section{Evolution of Bejan Number}

This evolution increases very fast in the first moments to stabilize towards a constant value. It observed that, regardless of the Prandtl number, the Bejan number is greater than $\mathbf{0 . 5}$, which shows that the creation of entropy is essentially due to temperature gradients. It shows the increase of Bejan number into the cavity by augmenting Prandtl number.

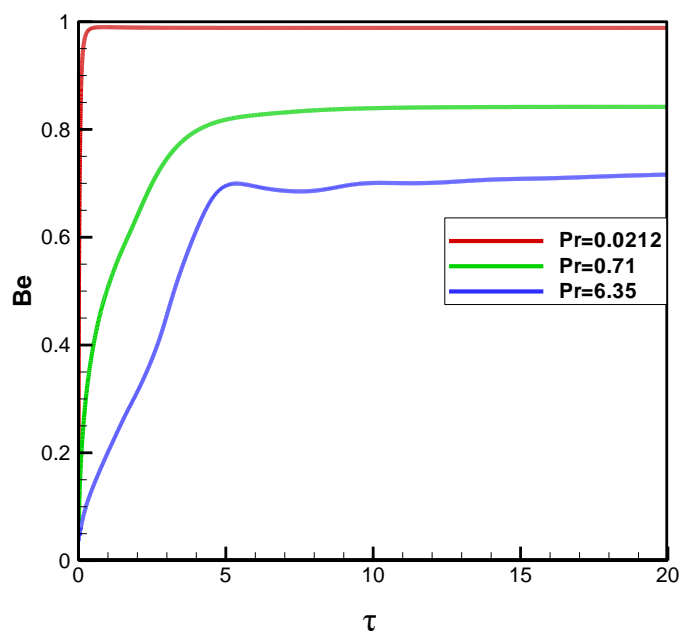

Figure 7. Evolution of Bejan number for different Prandtl numbers, $\mathrm{Ri}=1, \mathrm{Re}=100$ and $\gamma=10^{-4}$

\section{Evolution of Nusselt Number}

The heat transfer inside the square enclose is presented by average Nusselt numbers given by equation (12). It is found, for all values of the Prandtl number, a very fast decrease of $\mathrm{Nu}_{\mathrm{m}}$ in the first moments, then a tendency towards a limit value when the time increases. The increase in the number of Prandtl results in an increase in the average Nusselt number. 


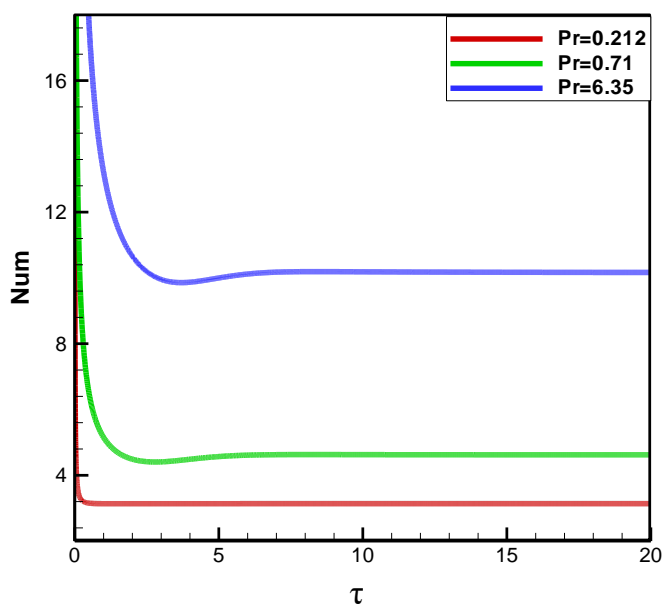

Figure 8. Evolution of average Nusselt number for different Prandtl numbers, $\mathrm{Ri}=1, \mathrm{Re}=100$ and $\gamma=10^{-4}$

\section{Effect of The Irreversibility Distribution Ratio on The Total Entropy Generation}

The evolution of the effect of irreversibility distribution ratio $\left(\gamma=10^{-2}\right.$ and $\left.10^{-4}\right)$ on the total entropy for different Prandtl number are plotted in figure 9 (a-b-c). It is observed that, for all Prandtl number, the total entropy generation increase by increasing the irreversibility distribution ratio and the increase of Prandtl number regardless the values of $\mathrm{Ri}=1$ and $\mathrm{Re}=100$ because of the increase of the velocity gradients.

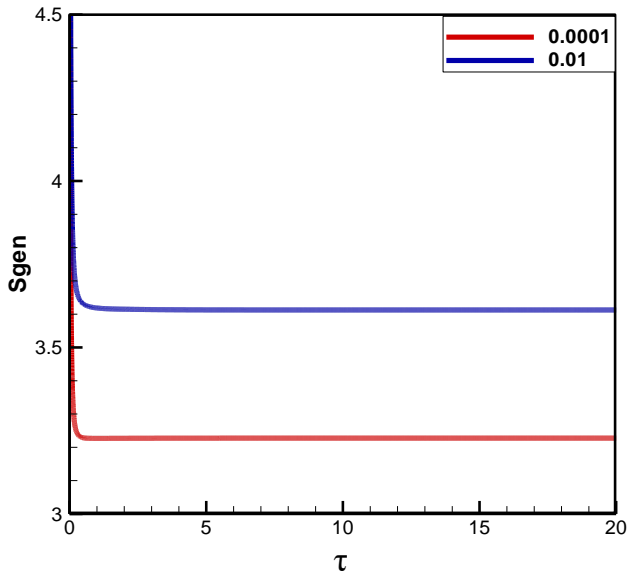

a) $\operatorname{Pr}=0.212$

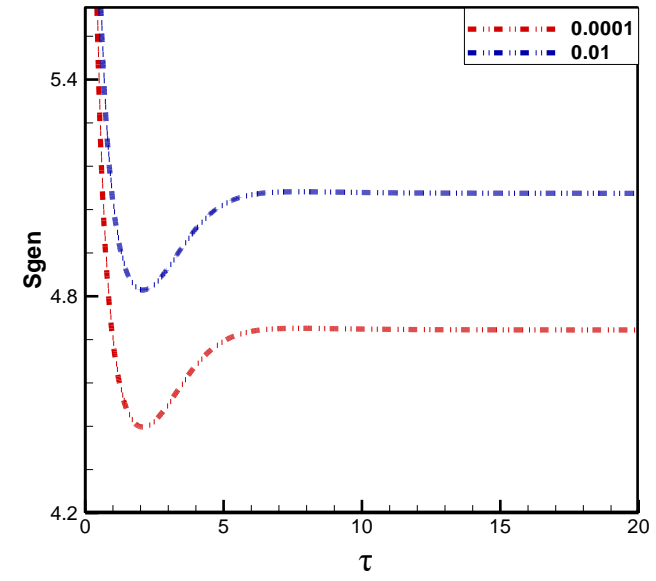

b) $\operatorname{Pr}=0.71$

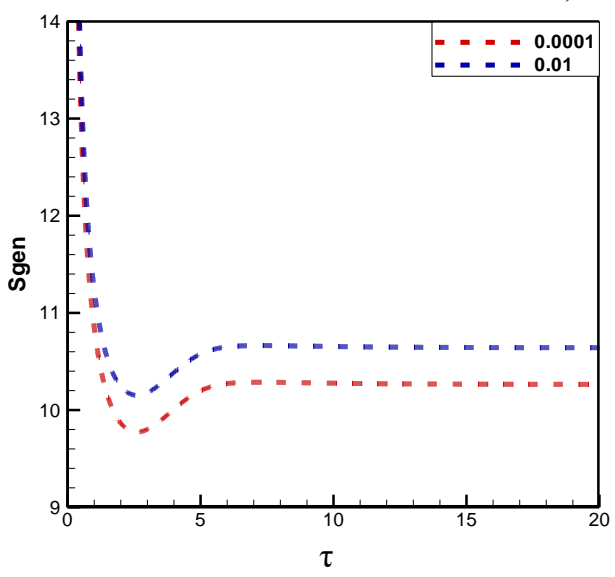

c) $\operatorname{Pr}=6.35$

Figure 9. Effect of the irreversibility distribution ratio on the total entropy generation for different Prandtl numbers, $\mathrm{Ri}=1, \mathrm{Re}=100$ and $10^{-2}<\gamma<10^{-4}$ 


\section{CONCLUSION}

In this numerical simulation of the Prandtl number effect on the entropy generation in laminar mixed convection, within a two dimensional square enclosure with moving cold sidewalls and heated from the bottom wall was investigated. The equations governing this phenomenon were discretized by the finite difference method, and the algebraic systems obtained were solved by appropriate numerical methods. The analysis of the preliminary results shows that the creation of the entropy increases with the increase of Pran dtl number (because of the increase of the velocity gradients) and the irreversibility distribution ratio, when this number increases $(\operatorname{Pr})$ the creation of the total entropy is essentially due to temperature gradients which is confirmed by the values of the Bejan number "Be $>0.5$ ".

As thermodynamic approach entropy generation in different form such as laminar mixed convection in a square fluid-filled cavity is critical process in many applications. It is discussed in this study to understand the response of the system which has heated wall with a constant temperature $T_{h}$ while the side-walls are maintained at a constant temperature $T_{c}$, and a constant velocity. Also it is seen that the irreversibility distribution ratio on the entropy generation in the cavity affected for the Prandtl number $=0.0212,0.71$ and 6.35 .

\section{NOMENCLATURE}

$\begin{array}{ll}C_{p} & \text { Specific heat, } \mathrm{kJ} / \mathrm{kg}{ }^{\circ} \mathrm{K} \\ g & \text { Gravitational acceleration, } \mathrm{m} \mathrm{s}^{-2} \\ \mathrm{k} & \text { Thermal conductivity, } \mathrm{W} / \mathrm{m}^{\circ} \mathrm{K} \\ l & \text { Length of the heated part, } \mathrm{m} \\ L & \text { Length of the cavity, } \mathrm{m} \\ P^{*} & \text { Pressure, } \mathrm{kg} \mathrm{m}^{-1} \mathrm{~s}^{-2} \\ P & \text { Dimensionless pressure } \\ P r & \text { Prandtl number } \\ \mathrm{Ri} & \text { Richard number } \\ S_{g e n} & \text { Entropy generation rate, } \mathrm{J} \mathrm{m}^{-3} \mathrm{~s}^{-1} \mathrm{~K}^{-1} \\ S_{g e n} & \text { Dimensionless local entropy generation } \\ t & \text { Time, sec } \\ T & \text { Temperature, } \mathrm{K} \\ T_{c} & \text { Cold temperature, } \mathrm{K} \\ T_{h} & \text { Hot temperature, } \mathrm{K} \\ u, v & \text { Velocity components along } x \text { and } y, \text { respectively, } \mathrm{m} \mathrm{s}^{-1} \\ U, V & \text { Dimensionless velocity components } \\ x, y & \text { Cartesian coordinates, } \mathrm{m} \\ X, Y & \text { Dimensionless coordinates }\end{array}$

Greek symbols

$\begin{array}{ll}\beta & \text { Thermal expansion coefficient, } \mathrm{K}^{-1} \\ \varepsilon & \text { Dimensionless length of the heated part } \\ \rho & \text { Density of a fluid. } \mathrm{kg} / \mathrm{m}^{3} \\ \theta & \text { Dimensionless temperature } \\ \tau & \text { Dimensionless temperature }\end{array}$

Subscripts

c Refers to cold

\section{REFERENCES}

[1] Philippe A. JAVET, Pierre LERCH, Eric PLATTNER. Introduction à la chimie pour ingénieurs, Deuxième Edition, Presses polytechniques et universitaires romandes, 1995.

[2] A. Bejan. A study of entropy generation in fundamental convective heat transfer, J. Heat Transfer, 101, 718-725, 1979. doi.org/10.1115/1.3451063 
[3] A. Bejan. The thermodynamic design of heat and mass transfer processes and devices, J. Heat and Fluid Flow, 8, 258-275, 1987. doi.org/10.1016/0142-727X(87)90062-2

[4] B. S. Yilbas. Natural Convection and Entropy Generation in a Square Cavity, International Journal of Entropy Research, Res, 22, 1275-1290, 1998. doi.org/10.1002/(SICI)1099-114X(199811)22:14<1275::AIDER453>3.0.CO;2-B

[5] A. C. Baytaç. Entropy generation for natural convection an inclined porous cavity, International Journal of Heat and Mass transfer, Vol 43, pp 2089-2099, 1999. doi.org/10.1016/S0017-9310(99)00291-4

[6] S. Z. Shuja, B. S. Yilbas, M. O. Iqbal. Mixed convection in a square cavity due to heat generation rectangular body, International Journal of Numerical Method for Heat \& Flow, Vol 10, pp 824-841, 2000. DOI: $10.1108 / 09615530010359120$

[7] B. S. Yilbas, S. Z. Shuja, M. O. Iqbal. Energy and entropy analysis in a square cavity with protruding body: effect of protruding body aspect ratio, International Journal of Energy Reserch, Vol 26, 851-866, 2002. doi.org/10.1002/er.824

[8] M. Magheribi, H.Abbassi, A. Ben Brahim. Entropy generation at the onset of natural convection, International Journal of Heat and Mass, Vol 46, pp 3444-3450, 2003. https://doi.org/10.1016/S0017-9310(03)00133-9

[9] G.E. Ovando-Chacon. Entropy generation due to mixed convection in an enclosure with heated corners, International Journal of Heat and Mass Transfer, Volume 55, Issue 4, pp 695-700, 2012. https://doi.org/10.1016/j.ijheatmasstransfer.2011.10.041

[10] Abd el malik Bouchoucha and a. Natural Convection And Entropy Generation Of Nanofluids In A Square Cavity, International Journal Of Heat And Technology,vol.33 No.4, pp.1-10, 2015. DOI: 10.18280/ijht.330401

[11] Fatih Selimefendigil, Hakan F. Oztop. Mixed convection and entropy generation of nanofluid flow in a vented cavity under the influence of inclined magnetic field, Technical Paper, pp1-12, 2019. DOI: 10.1007/s00542019-04350-1

[12] Almakki, M., Mondal, H., \& Sibanda, P. Entropy Generation in MHD Flow of Viscoelastic Nanofluids with Homogeneous-Heterogeneous Reaction, Partial Slip and Nonlinear Thermal Radiation. Journal of Thermal Engineering, 6(3), 327-345, 2020. DOI: 10.18186/thermal.712452

[13] Öğüt, E. B. Second Law Analysis Of Mixed Convection Of Magnetohydrodynamic Flow In An Inclined Square Lid-Driven Enclosure. Journal of Thermal Engineering, 5(6), 240-251, 2019. DOI: 10.18186/thermal.655023

[14] Karakurt, Sinan, and Umit Gunes. "A New Approach For Evaluating The Rankine Cycle Through Entropy Generation." Journal of Thermal Engineering 5, no. 6: 141-148. DOI: 10.18186/thermal.651508

[15] Rout, S. K. Experimental investigation and performance optimization of a cross flow heat exchanger by entropy generation minimization approach. Journal of Thermal Engineering, 5(2), 1-12, 2019. DOI: $10.18186 /$ thermal.519128

[16] Mansoor, S. Entropy Generation Rate In A Microscale Thin Film. Journal of Thermal Engineering, 5(5), 405413, 2019. DOI: $10.18186 /$ thermal.623211

[17] Ana-Maria Bianchi. Transferts thermiques, 1ère edition, Press polytechniques et universitaires romandes, 2004.

[18] Ana-Maria Bianchi, Transferts thermiques, 1ère ed, Press polytechniques et universitaires romandes.

[19] Clement Kleinstreuer. Modern Fluid Dynamics, Springer Verlag, USA, 2009.

[20] S. A. Gandjalikhan Nassab, A. Bahrami, R. Moosavi. Entropy generation in convection over an inclined backward-facing step with bleeding, International Journal of Science and Technology Education Research, Vol 2(5), pp 88-97, 2011. DOI: 10.1016/j.enconman.2007.10.031

[21] T. Kawamura, H. Takami, K. Kuwahara. New higher-order upwind scheme for incompressible Navier-Stokes equations, Numerical Method in Fluid Dynamics, Lecture Notes in Physics, vol, 218, pp. 291-295, 1985. DOI: $10.1007 / 3-540-13917-6 \_152$

[22] Dale A. Anderson, John C.Tannehill, Richard H. Plether. Computational Fluid Mechanics and Heat Transfer, Hemisphere Publishing Corporation, United states, 1984.

[23] John Wiley \& Sons. Applied Numerical methods, Copyright, by John Wiley \& Sons, 1962. 
[24] Menni, Y., Ghazvini, M., Ameur, H., Ahmadi, M. H., Sharifpur, M., \& Sadeghzadeh, M. Numerical calculations of the thermal-aerodynamic characteristics in a solar duct with multiple V-baffles. Engineering Applications of Computational Fluid Mechanics, 14(1), 1173-1197, 2000. Doi.org/10.1080/19942060.2020.18155886

[25] Ameur, H., Sahel, D., \& Menni, Y. Enhancement of the cooling of shear-thinning fluids in channel heat exchangers by using the V-baffling technique. Thermal Science and Engineering Progress, 100534, 2020. doi.org/10.1016/j.tsep.2020.100534

[26] Ameur, H., \& Menni, Y. Laminar cooling of shear thinning fluids in horizontal and baffled tubes: Effect of perforation in baffles. Thermal Science and Engineering Progress, 14, 100430, 2019. doi.org/10.1016/j.tsep.2019.100430

[27] Ameur, H., Sahel, D., \& Menni, Y. Numerical investigation of the performance of perforated baffles in a platefin heat exchanger. Thermal Science, (00), 90-90, 2020. DOI: 10.2298/TSCI190316090A

[28] Menni, Y., Ameur, H., Chamkha, A. J., Inc, M., \& Almohsen, B. Heat and mass transfer of oils in baffled and finned ducts. Thermal Science, 24(Suppl. 1), 267-276, 2020. DOI: 10.2298/TSCI20267M

[29] Laidoudi, H., \& Ameur, H. Investigation of the mixed convection of power-law fluids between two horizontal concentric cylinders: Effect of various operating conditions. Thermal Science and Engineering Progress, 20, 100731, 2020. doi.org/10.1016/j.tsep.2020.100731

[30] Aydin, O., \& Yang, W. J. Natural convection in enclosures with localized heating from below and symmetrical cooling from sides. International Journal of Numerical Methods for Heat \& Fluid Flow. Vo10, 5, pp.518-529, 2000. doi.org/10.1108/09615530010338196 